\title{
Optimization of Sample Preparation and Phloroglucinol Analysis of Marselan Grape Skin Proanthocyanidins using HPLC-DAD- ESI-MS/MS
}

\author{
N.-N. Liang ${ }^{1 \dagger}$, F. He ${ }^{1 \dagger}$, Q.-H. Pan ${ }^{1}$, J. Wang ${ }^{1}$, M.J. Reeves ${ }^{1,2}$, C.-Q. Duan ${ }^{1 *}$ \\ (1) Centre for Viticulture \& Enology, College of Food Science and Nutritional Engineering, China Agricultural University, \\ Beijing, 100083, China \\ (2) Faculty of Applied Science, Business and Computing, Eastern Institute of Technology, Napier 4142, New Zealand
}

Submitted for publication: September 2011

Accepted for publication: February 2012

Key words: flavan-3-ols, grape skins, HPLC-DAD-ESI-MS/MS, Marselan, phloroglucinol, proanthocyanidins

\begin{abstract}
Proanthocyanidins are a group of oligomeric or polymeric flavan-3-ols that are highly significant contributors to astringency in grapes and wines. An orthogonal L9(3) ${ }^{4}$ test was adopted to determine the optimal extraction conditions and acid-catalysis cleavage of proanthocyanidins in the presence of excess phloroglucinol. The qualitative and quantitative analyses were done using HPLC-DAD-ESI-MS/MS. The results showed that the maximum extraction was obtained using $0.3 \mathrm{~mol} / \mathrm{L} \mathrm{of} \mathrm{HCl}$ and $0.005 \mathrm{~g}$ of ascorbic acid with incubation at $70^{\circ} \mathrm{C}$ for $20 \mathrm{~min}$. The precision and accuracy of this method were acceptable. The composition of free flavan-3-ols and proanthocyanidins in the skins of 'Marselan' grapes (Vitis vinifera $\mathrm{L}$. cv.) was investigated. (-)-Epigallocatechin was found to be the most abundant free flavan-3-ol monomer and terminal subunits, whereas the extension subunits were mainly (-)-epicatechin-3-O-gallate in the early developmental stages, and primarily (-)-epigallocatechin and (-)-epicatechin in the middle and late stages.
\end{abstract}

\section{INTRODUCTION}

Proanthocyanidins, also known as condensed tannins, are a group of oligomers or polymers of flavan-3-ol units, which are synthesised via the flavonoid pathway (Dixon et al., 2005). As they provide grapes and wine with astringency, bitterness (Gawel, 1998; Peleg et al., 1999) and colour stability and ageing potential (Cheynier et al., 2006), proanthocyanidins are considered to be key determinants of wine quality (Singleton 1992; Dixon et al., 2005; Fulcrand et al., 2006). The influence of proanthocyanidins on grape and wine sensory quality is a function of their degree of polymerisation, composition and content (Fernández et al., 2007; Aron \& Kennedy, 2008). Flavan-3-ol monomers were found to be more bitter than oligomers or polymers (Peleg et al., 1999). Furthermore, although flavan-3-ol monomers, dimers and trimers have been found to taste more acid than astringent, oligomeric and polymeric proanthocyanidins give wine more marked astringency (Porter \& Woodruffe, 1984). In grape berries, proanthocyanidins, primarily accumulated in the skins, seeds and stems, are composed mainly of (+)-catechin, (-)-epicatechin, (-)-epigallocatechin and (-)-epicatechin-3-O-gallate, which are usually linked by $\mathrm{C} 4-\mathrm{C} 8$ and/or C4-C6 bonds (B-type), or additional C2-
O-C7 linkages (A-type) (Czochanska et al., 1980; Ricardo da Silva et al., 1991; Escribano-Bailón et al., 1992; Downey et al., 2003; Dixon et al., 2005). Grape variety, climate and vineyard management all affect the composition and content of flavan-3-ols (González-Neves et al., 2002; Kennedy et al., 2002; Cohen et al., 2008). It is essential and valuable to analyse and identify the composition units and their flavan3-ols content.

In recent years, much effort has been devoted to characterising the structure of proanthocyanidin oligomers and the composition of proanthocyanidin polymers. For several decades, high-performance liquid chromatography (HPLC) has been chosen for this analysis (Fulcrand et al., 1999; Labarbe et al., 1999; Bordiga et al., 2011). However, it has been difficult to satisfactorily resolve the exact profiles of proanthocyanidins accumulated in grape skins during berry development because of their structural complexity and diversity. In previous studies, benzyl mercaptan or toluene- $\alpha$-thiol were the most widely used trapping reagents during proanthocyanidin depolymerisation by acid-catalysis cleavage. Such thiol-based trapping agents are more effective nucleophiles than phloroglucinol trapping agents and they

${ }^{*}$ Corresponding author: Email: chqduan@yahoo.com.cn [Tel: +86 10 62737136; fax: +86 10 62737136].

tThese authors contributed equally to this work.

Acknowledgements: This research was supported by National Natural Science Foundation of China (grant no. 30871746 to C.-Q. Duan) and by the Specialized Research Fund for the Doctoral Program of Higher Education of China (No. 20090008110018). 
do not require ascorbic acid. There are some other trapping agents, such as cysteine or aminoethylthiol, which have been used in the analysis of proanthocyanidins (Torres \& Bobet, 2001; Torres et al., 2002). Recently, phloroglucinol, without the unpleasant smell of thiol-based trapping agents, has been used frequently as an advantageous trapping reagent (Rigaud et al., 1991; Guyot et al., 2001; Kennedy \& Jones, 2001). The extension subunits were released as the corresponding stable flavan-3-ol phloroglucinol adducts, and the terminal subunits were released as the free flavan-3-ol monomers without the phloroglucinol adducts. This greatly facilitates the analysis of the proanthocyanidin composition (Prieur et al., 1994; Kennedy \& Jones, 2001; Downey et al., 2003).

Although the method developed by Kennedy and Jones (2001) and Downey et al. (2003) has been used widely in the last ten years for sample preparation and the instrumental analysis of proanthocyanidins in grape skins, it is difficult and involved. We have developed an alternative method, which simplifies the treatment of the residues in particular. We also aimed to optimise the conditions of the extraction of the flavan-3-ol monomers, and the simultaneous extraction and acid-catalysis cleavage of grape skin proanthocyanidins in the presence of excess phloroglucinol. Simultaneously, we wanted to develop a rapid and sensitive method for the analysis of proanthocyanidin composition using a highperformance liquid chromatography-diode array detector (HPLC-DAD) linked to an electrospray ionisation tandem mass spectrometer (ESI-MS/MS). The tandem MS further facilitated the qualitative analysis of the flavan-3-ols and their derivatives in grape skins, improving the quantification that was done by the retention time method. By this means, the free flavan-3-ol monomer and proanthocyanidin in 'Marselan' grape skins during berry development were characterised qualitatively and quantitatively.

\section{MATERIALS AND METHODS \\ Samples}

In 2007, 'Marselan' ( $V$. vinifera L. cv.) berries were collected from the Sino-French Demonstration Vineyard in Hebei Province in northern China. The vineyard was established in 2000. This region has a continental monsoon climate with a mean daily temperature during berry development of $24^{\circ} \mathrm{C}$. The grape samples were collected at weekly intervals, from blooming on 1 June to commercial harvest on 15 September. Samples were collected according to the method described by Boulton et al. (1995). Three 100-berry samples were selected from at least seven 10-bunch selections at similar positions of 30 whole vine selections. The fresh samples were taken to the laboratory within two hours. The skins were stripped off by hand and then ground in liquid $\mathrm{N}_{2}$ using a mortar and pestle. After that the skin powders were freeze dried using a LGJ-10 freeze drier at $-50^{\circ} \mathrm{C}$ and then extracted as described below.

\section{Reagents and chemicals}

Standards for (+)-catechin, (-)-epicatechin, (-)-epigallocatechin, (-)-epicatechin-3-O-gallate and phloroglucinol were purchased from Sigma-Aldrich Co. (St. Louis, MO, USA). HPLC-grade acetonitrile, acetone, methanol and acetic acid were obtained from the Fisher Company
(Fairlawn, NJ, USA). Deionised water $(<18 \mathrm{M} \Omega$ resistance) was obtained from a Milli-Q Element water purification system (Millipore, Bedford, MA, USA). Ascorbic acid and all the other chemicals were purchased from Sigma-Aldrich Co., unless noted otherwise.

Extraction of free flavan-3-ol monomers from grape skins The extraction of free flavan-3-ol monomers from the grape skins was performed by using the method of Downey, Harvey and Robinson, with further modifications by Downey et al. (2003). Two main factors, liquid-solid ratio and the required number of times each sample needed to be extracted, were evaluated for their impact on the flavan-3-ol content extracted. The influence of the solid liquid ratio on extraction from the lyophilised powders was also investigated. Two levels, 1 and $2 \mathrm{~mL}$, of the extraction solution (acetone/ distilled $\mathrm{H}_{2} \mathrm{O}, 7: 3 \mathrm{v} / \mathrm{v}$ ) were mixed in a screw-top $10 \mathrm{~mL}$ Eppendorf tube with $0.1 \mathrm{~g}$ of the lyophilised skin powders to achieve a liquid-solid ratio of $1: 0.1$ or $2: 0.1$. Ascorbic acid $(0.005 \mathrm{~g})$ was added in advance to prevent oxidation. The mixture was shaken for 15 minutes and then centrifuged ( $8000 \mathrm{~g}, 15 \mathrm{~min}, 4^{\circ} \mathrm{C}$ ), and the supernatant was collected. The residue was extracted twice with the same volume of extraction solution, and the supernatants were pooled.

When determining the optimum number of extractions, we mixed $0.1 \mathrm{~g}$ of the dried skin powder with $1 \mathrm{~mL}$ of the extraction solution and $0.005 \mathrm{~g}$ of ascorbic acid powder. The mixture was shaken and centrifuged and the supernatants were decanted into a new tube. This was repeated several times so that the optimum number could be determined for complete extraction. The supernatants for each trial were pooled respectively. Aliquots of the pooled supernatants, each of $400 \mu \mathrm{L}$, were transferred to fresh tubes and dried rapidly with a stream of dry nitrogen at $30^{\circ} \mathrm{C}$. The dried samples were re-suspended in $200 \mu \mathrm{L}$ of methanol acidified with $1 \% \mathrm{HCl}$ and then neutralised with $200 \mu \mathrm{L}$ of sodium acetate $(200 \mathrm{mM}, \mathrm{pH} 7.5)$. These samples were then analysed by HPLC-MS. Each treatment was done in triplicate.

\section{Extraction of proanthocyanidins from grape skins and their acid cleavage}

We adopted an orthogonal L9 $(3)^{4}$ test design to screen the extraction conditions when determining the optimum proanthocyanidin extraction from the grape skins and to determine the completeness of the acid-degradation of proanthocyanidins. Proanthocyanidins are acid-cleaved in the presence of phloroglucinol into the terminal flavan-3-ol subunits and the extension subunit phloroglucinol adducts (Kennedy et al., 2000; Kennedy \& Jones, 2001; Downey et al., 2003). The four factors, each at three levels, were extraction time $(10,20,30 \mathrm{~min})$, extraction temperature $\left(50,60,70^{\circ} \mathrm{C}\right)$, the volume of $\mathrm{HCl}(0.1,0.2,0.3 \mathrm{~mol} / \mathrm{L})$ and the quantity of ascorbic acid $(0.005,0.01,0.02 \mathrm{~g})$. The four factors were chosen on the basis of both the preliminary results of the single-factor tests and the published literature in which the main factors influencing the yield and purity of the target compounds had been found (Kennedy \& Jones, 2001; Prieur et al., 1994). The grape skin proanthocyanidin analysis was done using $0.1 \mathrm{~g}$ of the lyophilised skin powders, which were mixed with $1 \mathrm{~mL}$ phloroglucinol buffer 
containing $0.5 \mathrm{~g}$ phloroglucinol, and $\mathrm{HCl}$ and ascorbic acid as required by the experimental design, and $10 \mathrm{~mL}$ methanol. The mixtures were incubated at the temperatures for the appropriate trial time. They then were neutralized with $1 \mathrm{~mL}$ of sodium acetate and centrifuged at $8000 \times g$ and $4^{\circ} \mathrm{C}$ for 15 $\min$. The residues were re-extracted twice and all the pooled supernatants were mixed. Each treatment was carried out in triplicate.

\section{Analysis of flavan-3-ols by HPLC-DAD-ESI-MS/MS}

The extracts obtained above were filtered through $0.22 \mu \mathrm{m}$ inorganic membranes and then analysed directly. Samples were run on an Agilent 1200 Series HPLC-DAD-ESI-MS/ $\mathrm{MS}$, and MS analysis was performed in the negative ionisation mode. The flavan-3-ol components were separated using a reverse phase column (Zorbax SB-C18, 250×4.6 mm, $5 \mu \mathrm{m}$ ) and binary gradient elution with mobile phases containing $0.2 \% \mathrm{v} / \mathrm{v}$ aqueous acetic acid (mobile phase A) and $4: 1$ acetonitrile: $0.2 \%$ acetic acid (mobile phase $\mathrm{B}$ ) at a flow rate of $1 \mathrm{~mL} / \mathrm{min}$ at $25^{\circ} \mathrm{C}$. The elution conditions were modified for the solvent B gradient as follows: $0 \mathrm{~min}, 10 \% ; 20 \mathrm{~min}$, $10 \%$; $30 \mathrm{~min}, 15 \%$; $40 \mathrm{~min}, 20 \%$; $50 \mathrm{~min}, 33 \%$; $55 \mathrm{~min}$, $40 \%$; $58 \mathrm{~min}, 100 \%$; $63 \mathrm{~min}, 100 \%$; $64 \mathrm{~min}, 10 \%$. Injection volumes were $25 \mu \mathrm{L}$ and the DAD detection wavelength was $280 \mathrm{~nm}$. The column was then re-equilibrated with $10 \% \mathrm{~B}$ for $5 \mathrm{~min}$ before the next injection. The ESI parameters were as follows: nebuliser pressure, $30 \mathrm{psi}$; dry gas flow, $10 \mathrm{~mL} /$ min; dry gas temperature, $325^{\circ} \mathrm{C}$; scan at $m / z$ 100-1000. All analyses were replicated twice.

\section{Qualitative and quantitative analyses of flavan-3-ol monomers and proanthocyanidin composition}

The flavan-3-ol monomers and the phloroglucinol adducts of the acid-cleaved terminal flavan-3-ol subunits and of the extension subunits were identified by their standards and their corresponding negative mode MS information.

The content of flavan-3-ol monomers was quantified from the standard curves prepared from the commercial standards for (+)-catechin, (-)-epicatechin, (-)-epigallocatechin and (-)-epicatechin-3-O-gallate.

Proanthocyanidin was analysed by acid cleavage, and the terminal flavan-3-ol subunits were released from the proanthocyanidin oligomers and polymers. The content of the terminal subunits was calculated by subtracting the content of the free monomers in the non-acid-cleaved sample from the total free monomers of the acid-cleaved sampled. The flavan-3-ol extension subunits were released as their corresponding flavan-3-ol phloroglucinol adducts (Kennedy et al., 2000; Kennedy \& Jones, 2001; Downey et al., 2003). The mean degree of polymerisation $(\mathrm{mDP})$ was calculated as the ratio of the total terminal plus the total extension subunits and divided by terminal subunits.

\section{Statistical analysis}

Statistical analyses were performed using SPSS 11.5 software (for Windows, license no. 30001359390). One-way analysis of variance (ANOVA) was applied to all samples to determine the significant difference at the 5\% level. The data are shown as means \pm standard deviation (SD) of the three independent extractions with two analytical replicates.

\section{RESULTS AND DISCUSSION}

\section{Effects of extraction variables}

For the extractable flavan-3-ol monomers there was no apparent difference in the composition of the four flavan3-ol monomers for the two liquid-solid ratios, 1:0.1 and 2:0.1, that were trialled (Table 1). The flavan-3-ol monomers detected in the skins of 'Marselan' grapes were $(+)$-catechin, (-)-epicatechin, (-)-epigallocatechin and (-)-epicatechin-3$O$-gallate. Therefore, a liquid:solid ratio of 1:0.1 was adopted in the following experiment.

The influence of the repeated extraction of the skin powders on the amount of flavan-3-ol monomers extracted is shown in Table 1. Flavan-3-ol monomers were extracted with repeated extraction, reaching a maximum after four extractions. However, there was no statistical difference between three and four extractions. Thus, we used three extractions in the following study.

The conditions used in acid-catalysis and cleavage of proanthocyanidins in the presence of plant tissue had a noticeable effect on the results from the purified proanthocyanidins. In particular, the addition of water affects the reaction dramatically (Bae et al., 1994; Kennedy \& Jones, 2001). Hence, a large amount of the proanthocyanidins remained in the residues after the extraction of proanthocyanidins from the grape skins when using acetone and water (7:3 v/v) (Downey et al., 2003). In order to avoid the influence of the water, we first lyophilised the grape skin sample to a dried powder and then simultaneously extracted and depolymerised the proanthocyanidins. This is similar to the post-extraction treatment of residues reported by Downey et al. (2003). However, we did not employ the solid phase extraction reported by Kennedy and Jones (2001), because it not only removes carbohydrates and organic acids, but also the low molecular weight monomeric flavan-3-ols, which are important to our research (Kennedy \& Jones, 2001).

After conducting the nine runs as determined by our L9 $(3)^{4}$ design, the $\mathrm{K}$ and $\mathrm{R}$ values were calculated for the screening of the optimal factors and concentration in order to optimise proanthocyanidin extraction and acid cleavage. The results are shown in Table 2. In terms of $\mathrm{R}$ value, the effects of the levels of the four factors, as trialled, on the extraction yield of proanthocyanidins from grape skins were, in decreasing order, extraction time (C) $>\mathrm{HCl}$ concentration (D) $>$ extraction temperature $(\mathrm{A})>$ ascorbic acid concentration (B). The effect of ascorbate concentration was found to be much smaller than that of the three other factors. From our k values, the optimal extraction conditions were determined to be: extraction time $20 \mathrm{~min}, 0.3 \mathrm{~mol} / \mathrm{L}$ $\mathrm{HCl}$, extraction temperature $70^{\circ} \mathrm{C}$ and $0.005 \mathrm{~g}$ ascorbic acid, being $\mathrm{C} 2, \mathrm{D} 3, \mathrm{~A} 3$ and $\mathrm{B} 1$ respectively. The higher temperature and acid level conditions are more severe than those used by Kennedy and Jones (2003). These conditions facilitated the extraction and cleavage reaction, but did not seem to result in undesirable oxidation, even in the present of plant tissues. The terminal subunit and the extension subunit concentrations in Marselan found by our method were quite different from those reported in other grape varieties, using the method reported previously, and are discussed below (Downey et al., 2003). 


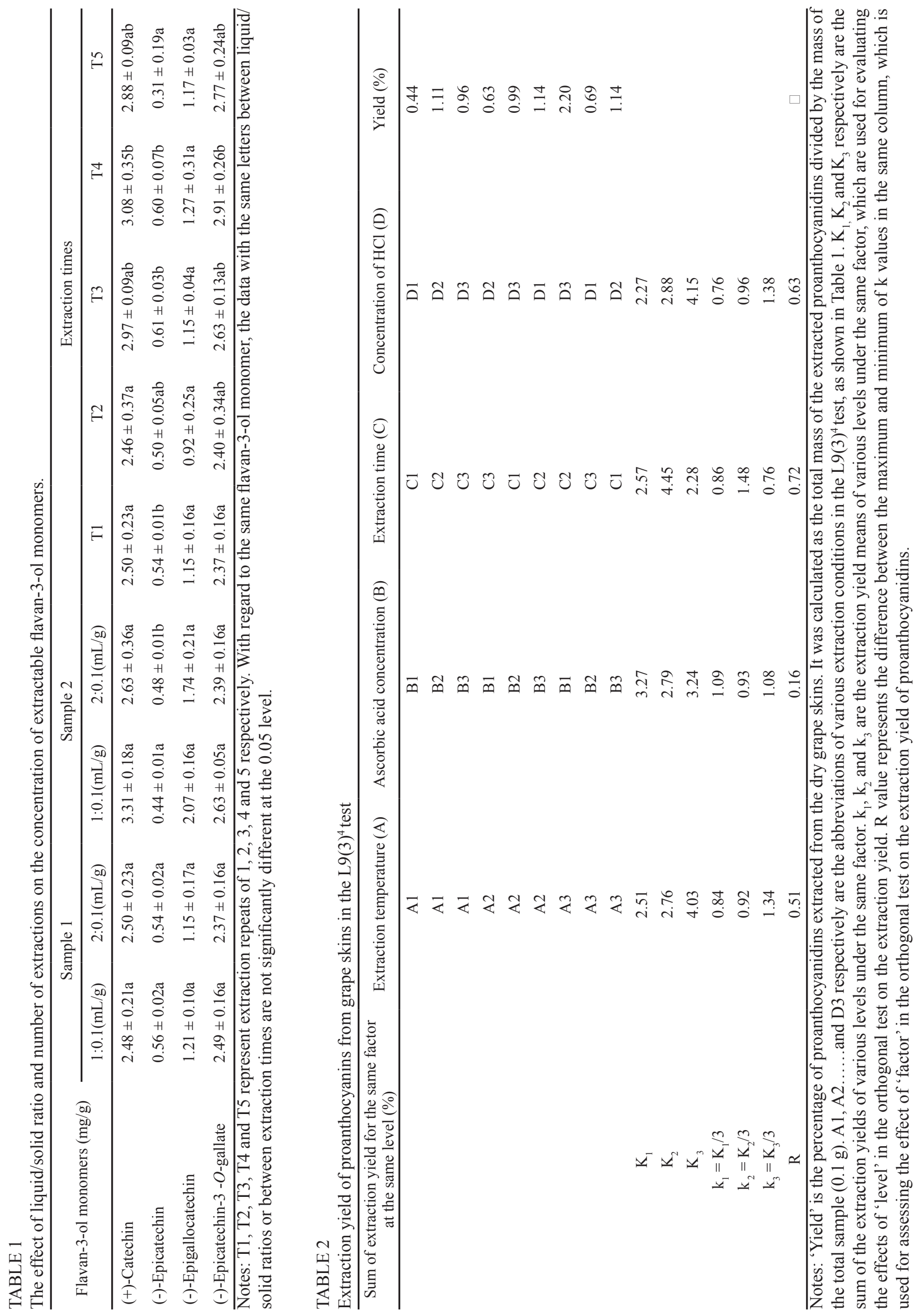




\section{Validation of the proposed extraction methods}

The accuracy of the proposed extraction method was estimated by using the percentage recovered. Three levels of a mixture of the standards containing four flavan-3ol monomers were added to the extraction solutions, and the extraction process for the flavan-3-ol monomers and proanthocyanidins from the skins was performed following the above method. The results are shown in Table 3. The recovery for various flavan-3-ol monomers was in range of $82 \%$ to $100 \%$, and the recovery for the extraction and acid cleavage of proanthocyanidins also exceeded $82 \%$, except in the case of (-)-epicatechin, which was only $74.9 \%$. We consider these recovery percentages to be acceptable. The precision of the proposed extraction methods was estimated using the relative standard deviations (RSD), expressed as the percentage of standard deviations to the mean content of the individual flavan-3-ols. Six independent extractions were carried out using the same grape skin samples as previously $(n=6)$. As can be seen from Table 3, the RSDs of individual flavan-3-ols were all less than 5\%, indicating that the two proposed extraction methods had good repeatability.

To determine the stability of the extracted and the acidtreated compounds, we analysed the same extract six times by HPLC-MS in one day and again within three days, and calculated the RSD. For the analysis of the extracted flavan3-ol monomers, the RSD of the retention time ranged from $0.010 \%$ to $2.190 \%$ in a day and from 0.096 to $0.458 \%$ within three days, while the RSD of peak area ranged from $0.400 \%$ to $3.790 \%$ for the same day and between 0.600 and $2.718 \%$ for analyses performed within three days. For the analysis of the phloroglucinol adducts from the acid cleavage of proanthocyanidins, the RSDs of the retention times ranged from $0.010 \%$ to $0.170 \%$ for the same day analyses and from $0.070 \%$ to $0.300 \%$ within three days, while the RSD of peak area ranged between $0.530 \%$ and $3.380 \%$ and between $1.780 \%$ and $3.150 \%$. These data indicate that quantitative analysis should be performed within a few days after the extraction, which is similar to previous reports (Muñoz et al., 2008). The calibration curve for each flavan-3-ol monomer was established by applying nine different blends of the standards (Table 4). Other parameters, such as linear range, correlation coefficient $(\mathrm{R})$ and limit of detection (LOD), are also listed in Table 4 . The results showed that the LOD of individual flavan-3-ol monomers was relatively low $(0.0561-0.2441 \mathrm{mg} / \mathrm{L})$, indicating that this HPLC-MS method has a high degree of sensitivity.

\section{Identification of flavan-3-ols and their corresponding phloroglucinol adducts from grape skins}

By using this reverse phase HPLC-MS method, flavan-3-ol monomers and their corresponding phloroglucinol adducts could be separated quickly and efficiently within 50 minutes, which is half the time of the commonly used methods of Kennedy and Jones (2001) and Downey et al. (2003), as shown in Fig. 1. In the present HPLC analysis of the flavan3 -ols and their phloroglucinol adducts, the retention time of these compounds changed slightly $(<0.1 \mathrm{~min})$ from time to time, although the elution order remained constant. The application of the tandem MS detector readily confirmed the identities. The four flavan-3-ol monomers, $(+)$-catechin, (-)-epicatechin, (-)-epigallocatechin and (-)-epicatechin-3$O$-gallate, could be identified easily according to their MS spectra and by comparison with their standards (Table 5) (Friedrich et al., 2000; Li \& Deinzer, 2007). The molecular ions of (+)-catechin [M-H] ${ }^{-}$and (-)-epicatechin [M-H] at $\mathrm{m} / \mathrm{z}$ 289 generated the fragment ions at $\mathrm{m} / \mathrm{z} 167,163$ and 137 by benzofuran-forming fission (BFF), heterocyclic fission (HRF) and the retro-Diels-Alder (rDA) fragmentation of the C-rings of the flavonoid skeleton. The molecular ions of (-)-epigallocatechin [M-H] at $\mathrm{m} / \mathrm{z} 305$ also produced fragment ions at $m / z 179,167$ and 137 by the same fission mechanisms. However, the molecular ion of (-)-epicatechin3-O-gallate $[\mathrm{M}-\mathrm{H}]^{-}$at $\mathrm{m} / \mathrm{z} 441$ was most likely split at the linkage of the acyl group to form fragment ions at $m / z 289$ and 169 as (-)-epicatechin and gallic acid ions respectively (Li \& Deinzer, 2007).

Besides the rDA fragmentation, quinonemethide (QM) cleavage of the 'interflavan linkage' between flavan-3-ol and the phloroglucinol moiety occurred more frequently than with the other two fission mechanisms (BFF and HRF) when the flavan-3-ol phloroglucinol adducts were analysed. Thus, (+)-catechin and (-)-epicatechin adducts

TABLE 3

Analysis of recovery and relative standard deviation (RSD) of four flavan-3-ol monomers by the developed extraction procedure

\begin{tabular}{lcccccc}
\hline Flavan-3-ols & $\begin{array}{c}\text { Initial content } \\
(\mathrm{mg} / \mathrm{g})\end{array}$ & $\begin{array}{c}\text { Added amount } \\
(\mathrm{mg} / \mathrm{L})\end{array}$ & $\begin{array}{c}\text { Recovery of } \\
\text { method 1 }(\%)\end{array}$ & $\begin{array}{c}\text { RSD of method 1 } \\
(\mathrm{n}=6, \%)\end{array}$ & $\begin{array}{c}\text { Recovery of } \\
\text { method 2 }(\%)\end{array}$ & $\begin{array}{c}\text { RSD of method 2 } \\
(\mathrm{n}=6, \%)\end{array}$ \\
\hline (+)-Catechin & 0.5414 & 25 & 87.9 & 2.1 & 113.0 & 4.0 \\
& 0.5414 & 50 & 88.2 & 1.4 & 88.2 & 3.4 \\
& 0.5414 & 100 & 88.1 & 2.3 & 82.3 & 2.9 \\
(-)-Epicatechin & 0.0781 & 25 & 92.1 & 3.7 & 103.3 & 1.5 \\
& 0.0781 & 50 & 100.0 & 1.4 & 83.9 & 2.2 \\
(-)-Epicatechin-3-O-gallate & 0.0781 & 100 & 99.7 & 2.5 & 74.9 & 0.6 \\
& 0.8751 & 25 & 88.8 & 1.1 & 101.1 & 1.0 \\
(-)-Epigallocatechin & 0.8751 & 50 & 92.2 & 1.0 & 109.9 & 0.6 \\
& 0.8751 & 100 & 87.5 & 0.6 & 108.8 & 0.8 \\
& 0.1778 & 25 & 82.6 & 3.1 & 91.9 & 3.6 \\
\end{tabular}

Notes: method 1 represents the extraction of flavan-3-ol monomers; method 2 represents the extraction and acid cleavage of proanthocyanidins. 
TABLE 4

Quantitative results of four flavan-3-ol monomers by HPLC

\begin{tabular}{lcccc}
\hline Flavan-3-ols & Linear range $(\mathrm{mg} / \mathrm{L})$ & Linear equation & Correlation coefficient & Limit of detection $(\mathrm{mg} / \mathrm{L})$ \\
\hline (+)-Catechin & $1.7969-500$ & $Y=32.5270 x+23.4525$ & 0.9999 & 0.0561 \\
(-)-Epicatechin & $1.0156-260$ & $Y=24.3600 x+10.0338$ & 0.9999 & 0.1270 \\
(-)-Epicatechin-3-O-gallate & $0.9765-250$ & $Y=22.8730 x-261.6000$ & 0.9990 & 0.2441 \\
(-)-Epigallocatechin & $1.9531-500$ & $Y=2.4995 x-8.3834$ & 0.9999 & 0.1221 \\
\hline
\end{tabular}

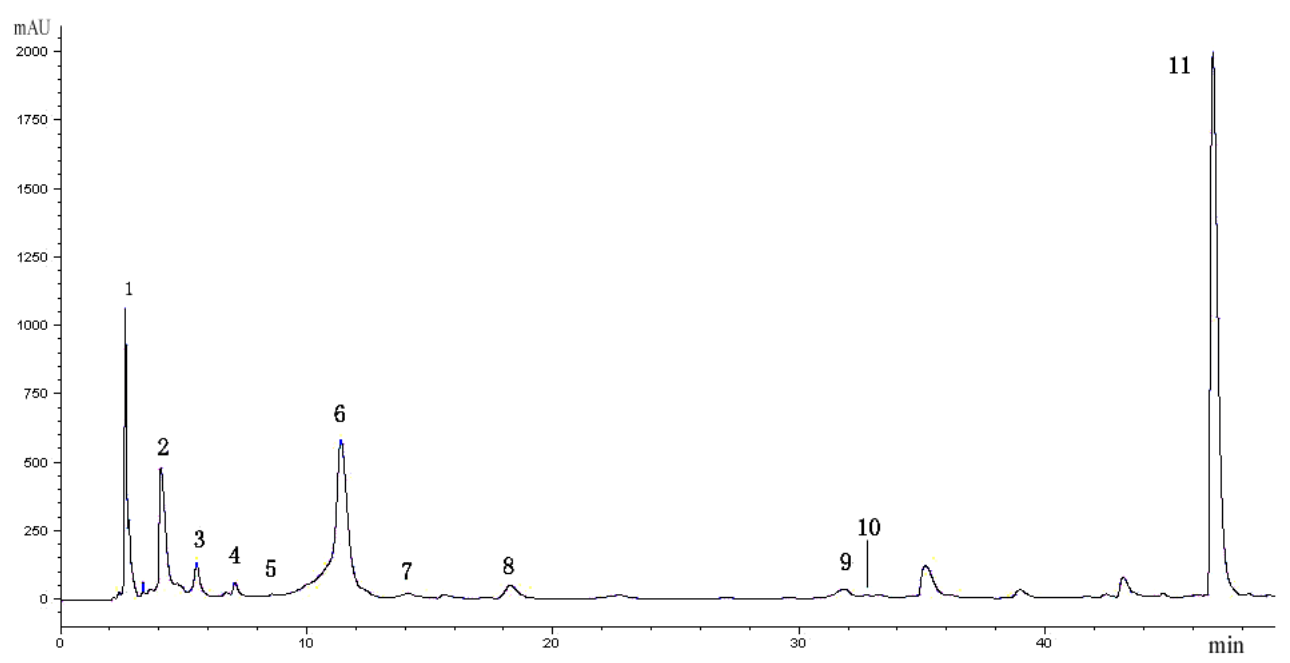

FIGURE 1

Reverse-phase HPLC chromatograms of flavan-3-ols and their corresponding phloroglucinol adducts after acid-catalysed cleavage of the proanthocyanidins in grape skins. Refer to Table 1 for the identification of each numbered peak.

TABLE 5

Flavan-3-ol monomers and their corresponding phloroglucinol adducts analysed by high-performance liquid chromatographydiode array detector-electronic ionisation-tandem mass spectrometry (HPLC-DAD-ESI-MS/MS)

\begin{tabular}{|c|c|c|c|}
\hline Peak & Retention time (min) & Compounds & {$[\mathrm{M}-\mathrm{H}]^{-}\left(\right.$Fragment-MS $\left.{ }^{2}\right)(\mathrm{m} / \mathrm{z})$} \\
\hline 1 & 2.786 & Ascorbic acid & $175(115)$ \\
\hline 2 & 4.053 & Phloroglucinol & $125(111)$ \\
\hline 3 & 5.562 & (-)-Epigallocatechin adduct & $429(303,261,167,125)$ \\
\hline 4 & 7.112 & Gallic acid & $169(143)$ \\
\hline 5 & 10.095 & $(+)$-Catechin adduct & $413(287,261,125)$ \\
\hline 6 & 11.362 & (-)-Epicatechin adduct & $413(287,261,125)$ \\
\hline 7 & 14.239 & (-)-Epigallocatechin & $305(261,179,167,137)$ \\
\hline 8 & 18.495 & $(+)$-Catechin & $289(245,167,163,137)$ \\
\hline 9 & 32.067 & (-)-Epicatechin-3-O-gallate adduct & $565(439,413,395,287,245,169)$ \\
\hline 10 & 32.905 & (-)-Epicatechin & $289(245,167,163,137)$ \\
\hline 11 & 46.904 & (-)-Epicatechin-3-O-gallate & $441(289,331,271,169)$ \\
\hline
\end{tabular}

that had the molecular ions $[\mathrm{M}-\mathrm{H}]^{-}$at $m / z 413$ could produce fragment ions at $m / z 261$ and 151 by rDA fission and could generate fragment ions at $m / z 287$ and 125 by QM cleavage. Correspondingly, the molecular ion of (-)-epigallocatechin adduct [M-H] ${ }^{-}$at $\mathrm{m} / \mathrm{z} 429$ could produce fragment ions at $\mathrm{m} / \mathrm{z}$ $303,261,167$ and 125 by similar fission mechanisms. The fragmentation of (-)-epicatechin-3-O-gallate adduct is a little more complicated. By QM cleavage between the C-ring and D-ring, its molecular ions [M-H] ${ }^{-}$at $m / z 413$ could generate fragment ions at $m / z 439$ and 125 directly. On the other hand, (-)-epicatechin-3-O-gallate adduct could produce fragment ions at $\mathrm{m} / \mathrm{z} 413$ and 169 , and the former could also undergo the further QM cleavage to create fragment ions at $m / z 287$. Furthermore, the fragment ion at $\mathrm{m} / \mathrm{z} 413$ could also generate fragment ions at $m / z 395$ and 245 by losing $\mathrm{H}_{2} \mathrm{O}$ or other compounds. Due to the lack of standards, the MS spectra of these flavan-3-ol phloroglucinol adducts, especially the molecular ions $[\mathrm{M}-\mathrm{H}]^{-}$and some of the specific fragment 
ions, proved to be useful for their identification, as shown in Fig. 2.

\section{Proanthocyanidin composition and its changes in the skins of developing 'Marselan' grapes}

These optimised methods were applied to the analysis of the proanthocyanidins in grape skins throughout berry development. The accumulation of the four different flavan3-ols is illustrated in Fig. 3, as the free monomers, the terminal subunits and the extension subunits.

On a per gram dry weight basis, the content of the total free flavan-3-ol monomers in grape skins reached its highest concentration in the first week after flowering, with a peak of $19.86 \mathrm{mg} / \mathrm{g}$, and then decreased sharply within four weeks after flowering. After that, the accumulation of flavan-3-ol monomers decreased slightly until commercial harvest at 15 weeks after flowering (Fig. 3A). This indicates that flavan-3-ol monomers initially accumulate in the early period of berry development. Although the biosynthesis of flavan-3-ols continues to véraison, no more free monomers would be accumulated at about eight weeks after flowering (Downey et al., 2003) because of the subsequent synthesis of proanthocyanidins. After véraison, the stream of flavonoid biosynthesis leads to the production of anthocyanins and other compounds (Muñoz et al., 2008), resulting in a decrease in free flavan-3-ol monomers.

In 'Marselan' grape skins, (-)-epigallocatechin was found to be the most abundant flavan-3-ol monomer, followed by (-)-epicatechin-3-O-gallate. The changes in (-)-epigallocatechin during berry development were consistent with those of total flavan-3-ol monomers, while (-)-epicatechin-3-O-gallate showed its highest content at the onset of flowering and then declined sharply to a low concentration. The contents of $(+)$-catechin and (-)-epicatechin were much lower than those of the two other monomers and both changed slightly during berry development (Fig. 3B). The present findings are different from those from some previous reports, on different grape varieties grown in different countries, in which $(+)$-catechin was always the predominant flavan-3-ol monomer, followed by (-)-epicatechin and (-)-epigallocatechin (Downey et al., 2003; Muñoz et al., 2008). Furthermore, (+)-catechin accumulated steadily from flowering and the content of $(+)$-catechin per berry reached a peak at around two weeks before véraison. On the other hand, the concentration of (-)-epicatechin-3-O-gallate monomer was low and remained relatively constant throughout berry development. In another report, no (-)-epigallocatechin and (-)-epicatechin-3-Ogallate monomers were detected in the grape skins (Muñoz et al., 2008). These differences suggest that the proportion of flavan-3-ol monomers varies greatly between different grape cultivars with, perhaps, some regional and possibly viticultural management influence.

Relatively high levels of terminal subunits and extension subunits were also observed in the first four weeks. The maximum content peak of total terminal subunits occurred at two weeks after flowering and that of total extension subunits in the first week (Fig. 3C and 1E). After that, the content of these composition subunits decreased and remained at a low level, which was similar to the trend of total free monomers. In this study, the most abundant terminal subunit was (-)-epigallocatechin throughout the whole berry development. As shown in Fig. 3C and 1D, the change in (-)-epigallocatechin in the developing grape berries overall was similar to the total terminal subunits. An exception was seen with (-)-epicatechin, which showed a sudden increase at two weeks after flowering, while (-)-epicatechin, (+)-catechin and (-)-epicatechin were all detected at low concentrations as the terminal subunits in 'Marselan' grape skins and remained almost unchanged during berry development (Fig. 3D). This
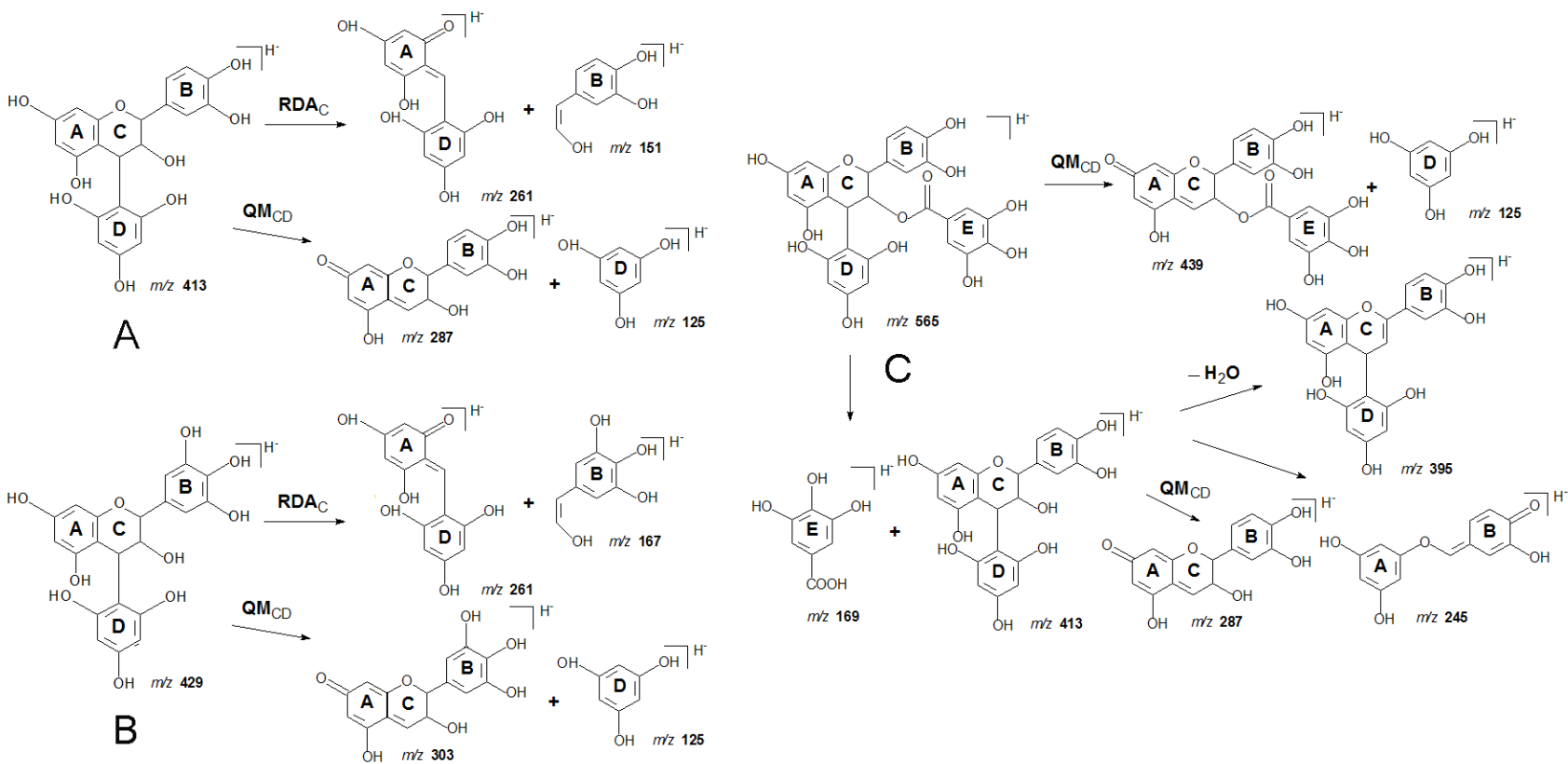

FIGURE 2

Characteristic fragmentation offlavan-3-ols phloroglucinol adducts. (A) (+)-Catechin or (-)-epicatechin; (B) (-)-Epigallocatechin; (C) (-)-Epicatechin-3-O-gallate. 


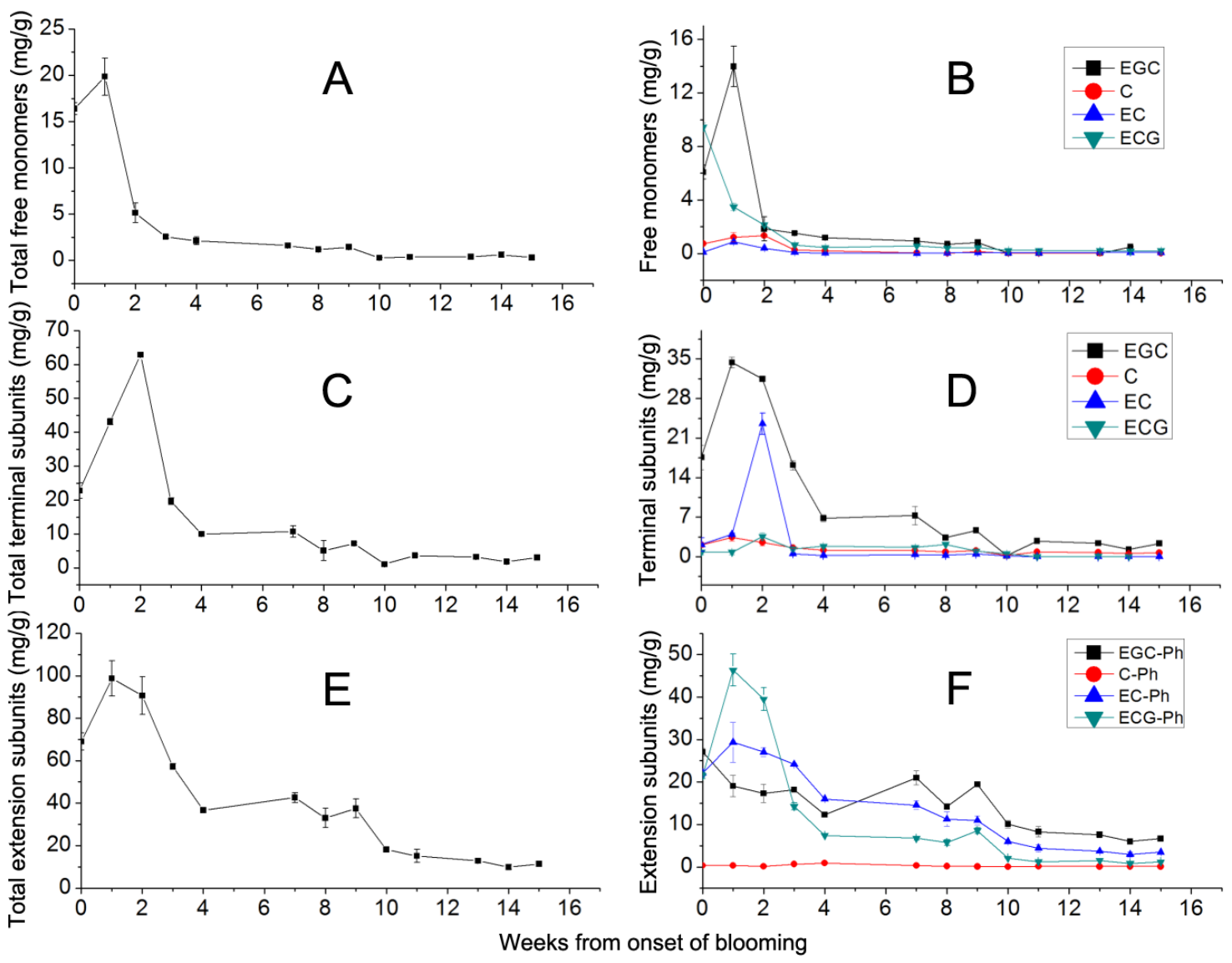

FIGURE 3

Changes to flavan-3-ol monomers and proanthocyanidin subunits in the skins of developing 'Marselan' grapes. (A) Total free monomers ( $\mathrm{mg} / \mathrm{g}$ dry weight of skin); (B) Free monomers (mg/g dry weight of skin); (C) Total terminal subunits (mg/g dry weight of skin); (D) Terminal subunits (mg/g dry weight of skin); (E) Total extension subunits (mg/g dry weight of skin); (D) Extension subunits ( $\mathrm{mg} / \mathrm{g}$ dry weight of skin). For each sample, three independent aliquots were extracted, cleaved and analysed by HPLC-DAD-ESI-MS/MS. The data presented are the mean $( \pm \mathrm{SE})$ of the triplicate extractions. Abbreviations used to represent these flavan-3-ols and their corresponding phloroglucinol adducts are as follows: (EGC) (-)-Epigallocatechin; (C) (+)-Catechin; (EC) (-)-Epicatechin; (ECG) (-)-Epicatechin-3-O-gallate; (EGC-Ph) (-)-Epigallocatechin-phloroglucinol adduct; (C-Ph) (+)-Catechin-phloroglucinol adduct; (EC-Ph) (-)-Epicatechin-phloroglucinol adduct; (ECG-Ph) (-)-Epicatechin-3-Ogallate-phloroglucinol adduct.

phenomenon was quite different from that found in previous studies, which showed that the composition of terminal subunits in the skins was primarily $(+)$-catechin, with low levels of (-)-epicatechin and (-)-epicatechin-3-O-gallate and almost no (-)-epigallocatechin observed (Monagas et al., 2003; Koerner et al., 2009). The reason for this difference is not clear, although it may be related to viticultural factors.

As for the extension subunits in 'Marselan' grape skins, their constituents differed from those of the free monomers and the terminal subunits. In the first two weeks after flowering, (-)-epicatechin-3-O-gallate was the most abundant extension subunit and its change was in good agreement with that of total extension subunits. Accompanying berry development, (-)-epicatechin and (-)-epigallocatechin become major extension subunits. Moreover, the amount of (-)-epigallocatechin as an extension subunit was more than (-)-epicatechin in the middle and late developmental stages, which corresponded with the decrease in the content of free (-)-epigallocatechin monomer. It is suggested that free (-)-epigallocatechin in the middle and late developmental stages participates in the polymerisation as extension subunits. The findings were also different from those of previous reports, in which (-)-epicatechin was the major extension subunit during berry development. In addition, we also observed that the level of (+)-catechin as extension subunits in 'Marselan' grape skins was low and remained relatively constant (Fig. 3F), which was also noted by Downey et al. (2003).

The change in proanthocyanidin mDP in 'Marselan' grape skins is shown in Fig. 4. Except that proanthocyanidin $\mathrm{mDP}$ increased sharply from 4 to 14 subunits in week 10 after flowering before falling back to about 4 in subsequent weeks, the mDP remained between 2 and 5 subunits during 'Marselan' grape development. This result is also quite different from those of some early investigations in Shiraz grape skins, where the proanthocyanidins mostly fluctuated between about 30 and 40 subunits, and declined slowly after véraison until reaching a low level of around 20 subunits at harvest (Kennedy \& Jones, 2001; Downey et al., 2003). The reported profile of mDP in Shiraz was relatively more 


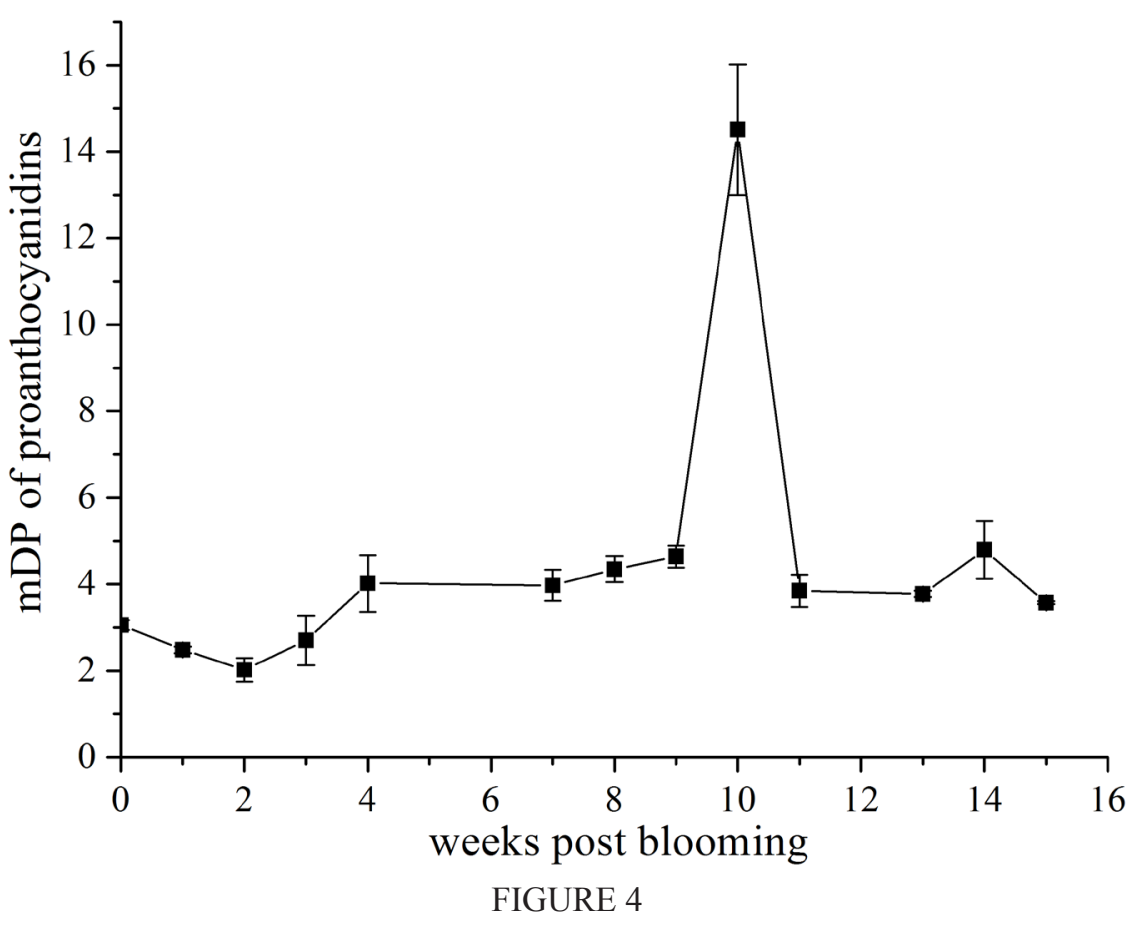

The mean degree of polymerisation (mDP) of proanthocyanidins in 'Marselan' grape skins throughout the whole berry development. The $\mathrm{mDP}$ was derived from the sum of terminal and extension subunits divided by terminal subunits.

constant than our result, and the mDP values were much higher than ours throughout berry development. As to the sudden change of mDP from 4 to 14 subunits at 10 weeks after flowing, the present result difficultly explained this point. The determination of $\mathrm{mDP}$ by phloroglucinolysis is limited due to the incompleteness of the cleavage reaction (Hanlin et al., 2011). To obtain accurate proanthocyanidin $\mathrm{mDP}$, additional information should be provided, such as yield conversion and assumed subunit composition, as well as inter-flavonoid linkages (Jorgensen et al 2004).

Differences in the grape varieties investigated, climatic factors, the year and viticultural practices might be partially responsible for the distinct differences in $\mathrm{mDP}$ values and trends in comparison with other published results. Our lower $\mathrm{mDP}$ values would result in our higher level of terminal subunits and lower level of extension subunits. We also converted and compared our results in 'Marselan' grape with those of the previous report on 'Shiraz' grapes in the same standard of measurement, namely the amount extracted per gram of grape skin fresh weight. The extension subunits in both studies were at similar levels, but the levels of the terminal units in our results were much higher than those in the previous report. Since the extension subunits were released as the flavan-3-ol phloroglucinol adducts and the terminal subunits were released as the free flavan-3-ol monomers without the phloroglucinol adducts, the extraction and reaction of proanthocyanidins in the grape skins should be sufficient. Thus, the differences might be due to the more efficient extraction and acid-catalysis cleavage of the proanthocyanidins in the samples, especially the low $\mathrm{mDP}$ oligomers.

\section{CONCLUSIONS}

In conclusion, we optimised the conditions for the extraction of the flavan-3-ol monomers, and the simultaneous extraction and acid-catalysis cleavage of proanthocyanidins in grape skins. By using HPLC-DAD-ESI-MS/MS, the composition of proanthocyanidins in the skins of 'Marselan' grapes was analysed simply and quickly. The results show that the composition of 'Marselan' proanthocyanidins and its various subunits differed significantly from those of other varieties. This might be the reason for the differences in astringency of different grape varieties, as well as their wines.

\section{LITERATURE CITED}

Aron, P.M. \& Kennedy, J.A., 2008. Flavan-3-ols: nature, occurrence and biological activity. Mol. Nutr. Food Res. 52, 79-104.

Bae, Y.S., Foo, L.Y. \& Karchesy, J.J., 1994. GPC of natural procyanidin oligomers and polymers. Holzforschung 48, 4-6.

Bordiga, M., Travaglia, F., Locatelli, M., Coïsson, J.D. \& Arlorio, M., 2011. Characterisation of polymeric skin and seed proanthocyanidins during ripening in six Vitis vinifera L. cv. Food Chem. 127, 180-187.

Boulton, R.B., Singleton, V.L., Bisson, L.F. \& Kunkee, R.E., 1995. Selection of state of ripeness for harvest and harvesting. In: Boulton, R.B. (ed). Principles and practices of winemaking. Chapman \& Hall, International Thomson Publishing, New York. pp. 52-60.

Cheynier, V., Dueñas-Paton, M., Salas, E., Maury, C., Souquet, J.-M., Sarni-Manchado, P. \& Fulcrand, H., 2006. Structure and properties of wine pigments and tannins. Am. J. Enol. Vitic. 57, 298-305.

Cohen, S.D., Tarara, J.M. \& Kennedy, J.A., 2008. Assessing the impact of temperature on grape phenolic metabolism. Anal. Chim. Acta 621, 57-67.

Czochanska, Z., Foo, L.Y., Newman, R.H. \& Porter, L.J., 1980. Polymeric proanthocyanidins. Stereochemistry, structural units, and molecular weight. J. Chem. Soc., Perkin Trans. 1, 2278-2286.

Dixon, R.A., Xie, D.-Y. \& Sharma, S.B., 2005. Proanthocyanidins - a final frontier in flavonoid research? New Phytol. 165, 9-28. 
Downey, M.O., Harvey, J.S. \& Robinson, S.P., 2003. Analysis of tannins in seeds and skins of Shiraz grapes throughout berry development. Aust. J. Grape Wine Res. 9, 15-27.

Escribano-Bailón, T., Gutiérrez-Fernández, Y., Rivas-Gonzalo, J.C. \& Santos-Buelga, C., 1992. Characterization of procyanidins of Vitis vinifera variety tinta del país grape seeds. J. Agric. Food. Chem. 40, 1794-1799.

Fernández, K., Kennedy, J.A. \& Agosin, E., 2007. Characterization of Vitis vinifera $\mathrm{L}$. Cv. Carménère grape and wine proanthocyanidins. J. Agric. Food Chem. 55, 3675-3680.

Friedrich, W., Eberhardt, A. \& Galensa, R., 2000. Investigation of proanthocyanidins by HPLC with electrospray ionization mass spectrometry. Eur. Food Res. Technol. 211, 56-64.

Fulcrand, H., Dueñas, M., Salas, E. \& Cheynier, V., 2006. Phenolic reactions during winemaking and aging. Am. J. Enol. Vitic. 57, 289-297.

Fulcrand, H., Remy, S., Souquet, J.-M., Cheynier, V. \& Moutounet, M., 1999. Study of wine tannin oligomers by on-line liquid chromatography electrospray ionization mass spectrometry. J. Agric. Food Chem. 47, 10231028 .

Gawel, R., 1998. Red wine astringency: a review. Austral. J. Grape Wine Res. 4, 74-96.

González-Neves, G., Gil, G. \& Ferrer, M., 2002. Effect of different vineyard treatments on the phenolic contents in Tannat (Vitis vinifera L.) grapes and their respective wines. Food Sci. Technol. Int. 8, 315-321.

Guyot, S., Marnet, N. \& Drilleau, J.-F., 2001. Thiolysis - HPLC characterization of apple procyanidins covering a large range of polymerization states. J. Agric. Food Chem. 49, 14-20.

Hanlin, R.L., Kelm, M.A., Wilkinson, K.L. \& Downey, M.O., 2011. Detailed characterization of proanthocyanidins in skin, seeds, and wine of Shiraz and Cabernet Sauvignon wine grapes (Vitis vinifera). J. Agric. Food. Chem. 59, 13265-13276.

Jorgensen, E.M., Marin, A.B. \& Kennedy, J.A. 2004. Analysis of the oxidative degradation of proanthocyanidins under basic conditions. J. Agric. Food Chem. 52, 2292-2296.

Kennedy, J.A. \& Jones, G.P., 2001. Analysis of proanthocyanidin cleavage products following acid-catalysis in the presence of excess phloroglucinol. J. Agric. Food Chem. 49, 1740-1746.

Kennedy, J.A., Matthews, M.A. \& Waterhouse, A.L., 2002. Effect of maturity and vine water status on grape skin and wine flavonoids. Am. J. Enol. Vitic. 53, 268-274.

Kennedy, J.A., Troup, G.J., Pilbrow, J.R., Hutton, D.R., Hewitt, D., Hunter, C.R., Ristic, R., Iland, P.G. \& Jones, G.P., 2000. Development of seed polyphenols in berries from Vitis vinifera L. cv. Shiraz. Aust. J. Grape Wine Res. 6, 244-254.
Koerner, J.L., Hsu, V.L., Lee, J. \& Kennedy, J.A., 2009. Determination of proanthocyanidin A2 content in phenolic polymer isolates by reversedphase high-performance liquid chromatography. J. Chromatogr. A 1216, 1403-1409.

Labarbe, B., Cheynier, V., Brossaud, F., Souquet, J.-M. \& Moutounet, M., 1999. Quantitative fractionation of grape proanthocyanidins according to their degree of polymerization. J. Agric. Food Chem. 47, 2719-2723.

Li, H.-J. \& Deinzer, M.L., 2007. Tandem mass spectrometry for sequencing proanthocyanidins. Anal. Chem. 79, 1739-1748.

Monagas, M., Gómez-Cordovés, C., Bartolomé, B., Laureano, O. \& Ricardo Da Silva, J.M., 2003. Monomeric, oligomeric, and polymeric flavan-3ol composition of wines and grapes from Vitis vinifera L. Cv. Graciano, Tempranillo, and Cabernet Sauvignon. J. Agric. Food Chem. 51, 6475-6481.

Muñoz, S., Mestres, M., Busto, O. \& Guasch, J., 2008. Determination of some flavan-3-ols and anthocyanins in red grape seed and skin extracts by HPLC-DAD: validation study and response comparison of different standards. Anal. Chim. Acta 628, 104-110.

Peleg, H., Gacon, K., Schlich, P. \& Noble, A.C., 1999. Bitterness and astringency of flavan-3-ol monomers, dimers and trimers. J. Sci. Food Agr. 79, 1123-1128.

Porter, L.J. \& Woodruffe, J., 1984. Haemanalysis: the relative astringency of proanthocyanidin polymers. Phytochemistry 23, 1255-1256.

Prieur, C., Rigaud, J., Cheynier, V. \& Moutounet, M. 1994. Oligomeric and polymeric procyanidins from grape seeds. Phytochemistry $36,781-784$.

Ricardo da Silva, J.M., Rigaud, J., Cheynier, V., Cheminat, A. \& Moutounet, M., 1991. Procyanidin dimers and trimers from grape seeds. Phytochemistry $30,1259-1264$

Rigaud, J., Perez-Ilzarbe, J., Ricardo da Silva, J.M. \& Cheynier, V., 1991. Micro method for the identification of proanthocyanidin using thiolysis monitored by high-performance liquid chromatography. J. Chromatogr. $540,401-405$.

Singleton, V.L., 1992. Tannins and the qualities of wines. In: Hemingway, R.W. \& Laks, P.E. (eds). Plant polyphenols. Plenum Press, New York. pp. $859-880$.

Torres, J.L. \& Bobet, R., 2001. New flavanol derivatives from grape (Vitis vinifera) byproducts. Antioxidant aminoethylthio-flavan-3-ol conjugates from a polymeric waste fraction used as a source of flavanols. J. Agric. Food Chem. 49, 4627-4634.

Torres, J.L., Lozano, C., Julià, L., Sánchez-Baeza, F.J., Anglada, J.M., Centelles, J.J. \& Cascante, M. 2002. Cysteinyl-flavan-3-ol conjugates from grape procyanidins. Antioxidant and antiproliferative properties. Bioorgan. Med. Chem. 10, 2497-2509. 